\title{
Motor cortical excitability behavior in chronic spinal cord injury neuropathic pain individuals submitted to transcranial direct current stimulation-case reports
}

\author{
Victor Gomide Carvalho $\mathbb{1}^{1} \cdot$ Rodrigo Lanna de Almeida $\mathbb{1}^{1,2} \cdot$ Raphael Boechat-Barros $^{3}$
}

Received: 3 July 2020 / Revised: 6 October 2020 / Accepted: 17 October 2020

(c) International Spinal Cord Society 2020

\begin{abstract}
Introduction Increased excitability of the motor cortex through transcranial direct current stimulation (tDCS) has been described as a non-pharmacological strategy for the treatment of Spinal Cord Injury neuropathic pain (SCINP). It is also believed that the ability to enhance motor cortex excitability (MCE) could be impaired within chronic SCINP individuals. The following case reports describe the MCE behavior in individuals with chronic SCINP submitted to electrical noninvasive neuromodulation.

Case presentations This article reports 11 cases with chronic SCINP in which each individual was submitted to a 5-day prepost MCE analysis in order to evaluate its behavior after the anodal tDCS sessions. All cases maintained ongoing pharmacological treatment. Four individuals have shown negative variation of the MCE, two of which reported pain intensity reduction. Three other individuals had MCE-positive variation along 5 days, from which only one reported a VAS 0.5 pain reduction after the 5th day of observation. The other four individuals did not present significant variation of the MCE.

Discussion The positive variation of MCE was significantly altered by adjunctive tDCS only in three individuals, though no clinically relevant reduction in pain intensity was reported among these participants. Key factors such as pain and injury duration, age, chronic medication use and underlying maladaptive neuroplasticity may influence responsiveness to brain stimulation within this population. These case reports try to add evidence for cautious recommendation of tDCS in chronic SCINP individuals and to the necessity of identifying groups of individuals that are most susceptible to neuromodulation.
\end{abstract}

\section{Introduction}

Spinal cord injury (SCI) is one of the most significant neurologic disability syndromes, with major physiological and psychological impacts, leading to a reduction in quality of life that requires great efforts in rehabilitation and lifelong management of secondary complications [1]. Among its main complications, pain of any kind is present in up to $80 \%$ of individuals [2]. In addition, the prevalence of neuropathic pain related to spinal cord injury (SCINP) is

Victor Gomide Carvalho

gomide80@gmail.com

1 Department of Spinal Cord Injury, SARAH Rehabilitation Hospital Network/SARAH Brasilia, Brasília, Brazil

2 Spinal Cord Injury Center, Heidelberg University Hospital, Heidelberg, Germany

3 Faculty of Medicine, University of Brasília, Brasília, Brazil estimated at $40-50 \%$ of the individuals [3, 4]. Among these, only $40 \%$ obtain significant improvement with adequate drug therapy [5], therefore it is considered a therapeutic challenge and has significant repercussions on disability.

One of the factors believed to influence the refractoriness of the pharmacological treatment of SCINP is the loss of central pain inhibitory mechanisms [6]. Maladaptive neuroplasticity phenomenon affects subcortical regions such as the thalamus, brainstem, and spinal cord and can be evidenced early (subacute) or years (chronic) after the spinal cord injury [7]. From a neurophysiological perspective, it is related to the loss of cortical inhibitory tonus from gammaaminobutyric acid pathways at the level of the spinal cord [8]. Both cortical and subcortical areas seem to have a functional connectivity with the motor cortex $[9,10]$.

Increased excitability of the motor cortex through transcranial direct current stimulation (tDCS) has been described as a non-pharmacological strategy for the treatment of pain by acting indirectly in areas of pain modulation such as the thalamus and subthalamic nuclei [11], by increasing 
downward inhibitory activity $[12,13]$. This technique has been tested in SCINP with favorable outcomes in pain intensity, although it is seemingly less effective within chronic SCI individuals [14]. Thus, the effectiveness of neuromodulation could rely on the potential excitability of the motor cortex, which can be measured by means of the variability of motor evoked potentials (MEP) elicited by cortical transcranial magnetic stimulation (TMS), in the following minutes after the stimulation [15]. Therefore, this measurable neurophysiologic phenomenon is an indirect evaluation of how the motor cortex changes over time after a given stimulus. However, this potential excitability behavior hasn't been tested in chronic SCINP individuals to the present date. Identifying a group of individuals susceptible to neuromodulation could help in the decisionmaking process, as with deciding whether to offer tDCS as adjuvant therapy.

This article reports the behavior of motor cortical excitability before and after cortical stimulus in eleven individuals, in which the use of consecutive anodal tDCS was performed in a SCINP management clinic.

\section{Case Presentations}

We present eleven cases of individuals with SCI and chronic NP, that were regularly followed at a specialized rehabilitation outpatient pain clinic at the Spinal Cord Unit at the Sarah Network of Rehabilitation Hospitals - Brasília, and were already enrolled for adjunctive pain therapy with anodal tDCS at the hospital's Neurophysiology Unit. All cases were invited to participate in the study between September and November 2018 if they met the following inclusion criteria: age equal to or above 18 years, SCI of any etiology and neuropathic pain as the most significant type of pain, time from injury and pain duration over 1 year, level of injury below T1 (in order access preserved contraction of the abductor digitalis minimus), being in use of at least one class of pain control medications (tricyclic antidepressants and/or anticonvulsants and/or opioids), pain intensity greater than or equal to 4 on the visual analog pain scale (VAS). Exclusion occurred if there was any contraindication for tDCS or TMS. such as a history of loss of consciousness or post-traumatic amnesia at the time of trauma, bony failure of the skull cap, epilepsy, implanted devices for treatment of previous pain, cardiac pacemakers or implantable defibrillators. Exclusion also occurred if there were any clinical findings that could make it difficult to evaluate cortical excitability, such as progression of spinal injury, uncontrolled and/or untreated psychiatric disorders, significant cognitive deficit, use or abuse of illicit drugs, spinal surgical procedures for treatment of prior pain, or other surgical procedures that could make it impossible
Table 1 Clinical characteristics.

\begin{tabular}{|c|c|}
\hline & Mean in years $(S D)$ \\
\hline Age & $42( \pm 13.3)$ \\
\hline Time since injury/pain & $6( \pm 3.9)$ \\
\hline \multirow[t]{2}{*}{ Baseline VAS score } & $6( \pm 1.2)$ \\
\hline & $n(\%)$ \\
\hline Sex (male) & $7(63.6)$ \\
\hline \multicolumn{2}{|l|}{ SCI etiology } \\
\hline Vehicle accident & $3(28)$ \\
\hline Falls & $1(9)$ \\
\hline Gun shot trauma & $4(36)$ \\
\hline Other traumatic & $1(9)$ \\
\hline Non-traumatic & $2(18)$ \\
\hline \multicolumn{2}{|l|}{ SCI classification (AIS) } \\
\hline Motor complete (A-B) & $9(82)$ \\
\hline Motor incomplete (C-D) & $2(18)$ \\
\hline \multicolumn{2}{|l|}{ Pain location } \\
\hline Below level SCINP & $5(45,5)$ \\
\hline At level SCINP & $5(45,5)$ \\
\hline Below and At Level SCINP & $1(9)$ \\
\hline \multicolumn{2}{|l|}{ Medication* } \\
\hline Gabapentinoids & $11(100)$ \\
\hline Antidepressants** & $8(73)$ \\
\hline Opioids & $2(18)$ \\
\hline Muscle relaxants (baclofen) & $1(9)$ \\
\hline Benzodiazepines & $1(9)$ \\
\hline
\end{tabular}

AIS - American Spinal Cord Injury Impairment Scale; VAS - Visual Analog Scale.

*participants could be in use of more than one class of medication.

**tricyclic or serotonin-norepinephrine reuptake inhibitors.

to evaluate the motor evoked potential (eg, nerve transfer procedure). The use of alternative complementary medicine within one month prior to the start of the study also precluded participation. Participants were asked to make sure they were able to attend the neurophysiology service for 5 consecutive days. All participants provided free and informed consent to participate in the research approved by the local Research Ethics Committee.

For each case, demographic and inherent characteristics of SCI and of the NP syndrome were collected from the patient's records. Group characteristics are described in Table 1. The neurological status is described according to the International Standards for Neurological Classification of SCI (ISNCSCI), and the classification of NP is described according to the recommendation of the International Spinal Cord Injury Pain (ISCIP) Classification [16, 17]. Other clinical and demographic data were collected at baseline: age, gender, level of injury, duration of pain, pain intensity and etiology of injury. The clinical and demographic data are described in Table 2. 
Table 2 Individual characteristics x MEP-quotient variation direction.

Participant Gender Age (y) ISNCSCI Pain duration (y) Medication (mg/day) VAS D1_resting VAS D5_30 min ISCIP classification

Individuals with positive variation of the MEP-quotient

$\begin{array}{lllllllll}2 & \text { F } & 32 & \text { AIS-B } & 6.4 & \text { Gabapentin } 600 \mathrm{mg} & 4 & 3,5 & \text { Bellow-level } \\ 6 & \text { F } & 45 & \text { AIS-B } & 15.4 & \begin{array}{l}\text { Pregabalin } 300 \mathrm{mg} ; \\ \text { Duloxetine } 60 \mathrm{mg}\end{array} & \text { Bellow-level } \\ 8 & \text { F } & 53 & \text { AIS-A } & 7.5 & \begin{array}{l}\text { Gabapentin 3600 mg; } 6 \\ \text { Amitriptyline 50 mg }\end{array} & \text { Bellow-level }\end{array}$

Individuals with negative variation of the $M E P$-quotient

\begin{tabular}{|c|c|c|c|c|c|c|c|c|}
\hline 1 & M & 29 & AIS-A & 2.4 & $\begin{array}{l}\text { Gabapentin } 1600 \mathrm{mg} \\
\text { Lamotrigine } 75 \mathrm{mg}\end{array}$ & 4 & 2 & Bellow-level \\
\hline 5 & M & 38 & AIS-B & 2.4 & $\begin{array}{l}\text { Gabapentin } 1500 \mathrm{mg} \\
\text { Amitriptyline } 25 \mathrm{mg}\end{array}$ & 6 & 6 & At-level \\
\hline 9 & M & 23 & AIS-B & 2.2 & $\begin{array}{l}\text { Gabapentin } 2400 \mathrm{mg} \\
\text { Nortriptyline } 50 \mathrm{mg} \\
\text { Metadon } 5 \mathrm{mg}\end{array}$ & 8 & 0 & At-level \\
\hline 11 & M & 31 & AIS-A & 7.5 & $\begin{array}{l}\text { Gabapentin } 2400 \mathrm{mg} \\
\text { Duloxetine } 60 \mathrm{mg}\end{array}$ & 8 & 8 & Bellow-level \\
\hline \multicolumn{9}{|c|}{ Individuals with $\mathrm{NO}$ variation of the $M E P$-quotient } \\
\hline 3 & $\mathrm{~F}$ & 49 & AIS-B & 5.2 & $\begin{array}{l}\text { Pregabalin } 300 \mathrm{mg} \\
\text { Venlafaxine } 150 \mathrm{mg} \\
\text { Tizanidine } 12 \mathrm{mg} \\
\text { Baclofen } 80 \mathrm{mg}\end{array}$ & 5 & 0.5 & Bellow-level \\
\hline 4 & M & 56 & AIS-B & 8.5 & $\begin{array}{l}\text { Gabapentin } 2000 \mathrm{mg} \\
\text { Amitriptyline } 25 \mathrm{mg}\end{array}$ & 10 & Drop-out & At-level \\
\hline 7 & M & 40 & AIS-D & 3.0 & $\begin{array}{l}\text { Pregabalin } 600 \mathrm{mg} \\
\text { Lamotrigine } 200 \mathrm{mg} \\
\text { Amitriptyline } 75 \mathrm{mg} \\
\text { Tramadol } 50 \mathrm{mg} \\
\text { Methadone } 10 \mathrm{mg}\end{array}$ & 8 & 6 & At-level \\
\hline 10 & M & 67 & AIS-D & 8.5 & Gabapentin $1200 \mathrm{mg}$ & 10 & Drop-out & At-level \\
\hline
\end{tabular}

ISNCSCI - International Standards for Neurological Classification of SCI.

AIS - ASIA Impairment Score.

ISCIP - International Spinal Cord Injury Pain Classification.

VAS - Pain Visual Analog Scale.

Each individual was submitted to a five-day pre-post MEP analysis in order to evaluate behavior after the tDCS sessions, as described below.

\section{Location of the point for stimulus and TMS pulses}

Each patient was positioned in the seated position, the chosen point for cortical stimulation (tDCS) and TMSinduced MEP was the primary motor cortex (M1). The chosen hemisphere was based on pain lateralization. For individuals with asymmetric pain, we used the contralateral M1, and for individuals with symmetric pain, we used the dominant, left (for right-handed patients) hemisphere [18]. The motor area was localized by means of TMS neuronavigation using a standard computer model with manual adjustments of individual anatomical references before each session (nasum, orbix, bilateral tragus) (Axilum Robotics TMS-Robot, http://www.axilumrobotics.com).
The location of interest corresponded to effective and perceptible contraction of the abductor of the minimum finger. The intensity was adjusted to corresponded to the MEP of best amplitude $(\sim 1 \mathrm{mV})$ and maintained the same throughout all the 5 consecutive days. The site was marked with a skin marker to ensure that the anode-tDCS stimulus would be delivered to the same area throughout the experiment.

\section{Measures of cortical excitability}

TMS was applied with an eight-shaped coil device (Magaven MagPro X10; http://www.magventure.com). Single-pulses of TMS were performed over the motor area correspondent to the contralateral hand, which was the same area in which tDCS was applied. MEP were recorded with an electromyographic surface electrode placed on the abductor muscle of the small finger, measured by the peak- 
to-peak amplitude automatically generated by the embedded computer software used for the electromyographic register (Brainsight Version 2.3.12). The subjects were instructed to relax. Serial MEP's were obtained before and after the tDCS sessions, and changes in cortical excitability were assessed by peak-to-peak amplitude measurements of these potentials [19]. Single-pulse TMS was delivered to obtain 20 MEPs as resting measures (pre-stimulus) in each day of the protocol. Five post-stimulus MEPs were obtained at the 1 st minute after the stimulus, then every $5 \mathrm{~min}$ until $30 \mathrm{~min}$ post-tDCS.

\section{Pain measurement}

Pain intensity was measured at rest, and immediately and 30 minutes post-tDCS, using the visual analog scale for pain (VAS).

\section{Motor cortex stimulation (tDCS)}

Anodal stimulation was performed over the motor cortical representation (M1) localized as mentioned above. The stimulus was delivered with an electrode size of $5 \times 7 \mathrm{~cm}$ $\left(35 \mathrm{~cm}^{2}\right)$ embedded in a saline solution for 20 min with a current of $2 \mathrm{~mA}$. The cathodic electrode was placed in the contralateral supraorbital region [18]. DC stimulator (NeuroConn DC-Stimulator; http://www.neurocaregroup. com) was used in all participants. Five sessions of neuromodulation with tDCS were performed on 5 consecutive days, keeping the tDCS settings unchanged in all sessions, according to the hospital's therapeutic neuromodulation protocol, which is based on previous publications $[14,20]$.

The entire evaluation took place at Neurophysiology Unit at the Sarah Network of Rehabilitation HospitalsBrasília.

\section{Statistical analysis}

The mean value was calculated for all the time-point measures - resting (pre-tDCS) and 1, 5, 10, 15, 20, 25, $30 \mathrm{~min}$ post-tDCS stimulus for each of the 5 days of the experiment (D1, D2, D3, D4, and D5). The mean values were then normalized by using the mean MEP at rest (pre-tDCS) for each participant. All values were therefore given as post/ pre-tDCS quotient (MEP-quotient), as done by Nitsche and Paulus in a landmark study in 2001 [15].

The Friedman test was applied to compare MEP-quotient measures throughout all 5 five days, using resting_D1 as a denominator, for each participant separately. Wilcoxon post-hoc test was performed whenever needed to determine the points of difference between-days $(\mathrm{D} 1 \times \mathrm{D} 2$; D1 $\times \mathrm{D} 3$; D1 $\times$ D4; D1 $\times$ D5).
For the purpose of analysis, only the worst pain was considered for analysis, whenever the participant related two different types of pain.

Significance difference was established at 5\% $(p<0.05)$.

\section{Results}

Table 1 shows the characteristics of the evaluated group of individuals. No adverse effect related to tDCS or TMS was reported by the participants, although two participants reported neuropathic pain crisis after the second day of the study, one related to urinary tract infection and another due to stressful family discussion and opted to interrupt tDCS therapy before initiating the third day procedures. One patient reported quality of sleep improvement. Table 2 summarizes the clinical characteristics of individuals and pain responses to the stimulation.

Overall seven participants were found to have significant differences in the between-days MEP-quotient analysis for repetitive measures (Table 3). Figure 1 outlines the variation in the post hoc day differences whenever the Friedman test demonstrated a significant difference among repeated measures.

Four individuals have shown negative variation in the MEP-quotient, two of which reported pain intensity reduction. Other three individuals had positive variation of the MEP-quotient along 5 days, from which only one reported a VAS 0.5 pain reduction after the fifth day of observation.

\section{Discussion}

The variation of motor-evoked potentials was significantly altered by therapeutic non-invasive neuromodulation in seven individuals, though no clinically relevant reduction in pain intensity was reported among these participants. Only three participants responded with a positive variation between-days on MEP measures, not accompanied by subjective pain response, and other four negatively (lower MEPs compared to baseline).

These findings could ratify the hypothesis that this population has a less excitable cortex than healthy individuals. It also suggests that there may be maladaptive neuroplasticity in these individuals due to chronicity of pain, although it is not possible to rule out the influence of chronic neurological dysfunction or chronic medication use.

The role of chronic pain in maladaptive plasticity is widely reported in other studies. In a meta-analysis conducted by Parker et al. [10], which evaluated various measures of cortical excitability in different types of chronic pain compared to healthy people, the reduction in intracortical inhibition measures (silent period and short-interval intracortical 
Table 3 Individual between-days MEP-quotient repeated measures analysis.

\begin{tabular}{|c|c|c|c|c|}
\hline Participant & Day & Median (IQR) & $p$ value & Post hoc $p$ value \\
\hline \multirow[t]{5}{*}{1} & D1 & $1.05(1 ; 1.18)$ & \multirow[t]{5}{*}{$0.00 *$} & $\mathrm{D} 1 \times \mathrm{D} 20.02 *$ \\
\hline & D2 & $0.9(0.83 ; 1)$ & & $\mathrm{D} 1 \times \mathrm{D} 30.09 *$ \\
\hline & D3 & $0.96(0.9 ; 1.06)$ & & $\mathrm{D} 1 \times \mathrm{D} 40.01 *$ \\
\hline & D4 & $0.86(0.71 ; 0.97)$ & & D1 × D5 $0.01 *$ \\
\hline & D5 & $0.58(0.72 ; 0.98)$ & & \\
\hline \multirow[t]{5}{*}{2} & D1 & $1.03(0.82 ; 1.55)$ & \multirow[t]{5}{*}{$0.02 *$} & $\mathrm{D} 1 \times \mathrm{D} 20.01^{*}$ \\
\hline & D2 & $4.92(2.39 ; 7.84)$ & & D1 × D3 0.26 \\
\hline & D3 & $2.65(0.69 ; 4.76)$ & & $\mathrm{D} 1 \times \mathrm{D} 40.05^{*}$ \\
\hline & D4 & $2.16(1.31 ; 3.29)$ & & D1 × D5 $0.01 *$ \\
\hline & D5 & $3.76(3.05 ; 4.86)$ & & \\
\hline \multirow[t]{5}{*}{3} & D1 & $1.16(1 ; 1.48)$ & \multirow[t]{5}{*}{0.5} & - \\
\hline & D2 & $1.28(0.98 ; 1.81)$ & & \\
\hline & D3 & $1.08(0.67 ; 1.08)$ & & \\
\hline & D4 & $0.98(0.66 ; 1.15)$ & & \\
\hline & D5 & $1.02(0.7 ; 1.41)$ & & \\
\hline \multirow[t]{5}{*}{$4 * *$} & D1 & $0.82(0.78 ; 1.01)$ & \multirow[t]{5}{*}{0.16} & - \\
\hline & D2 & $1.1(0.94 ; 1.26)$ & & \\
\hline & D3 & - & & \\
\hline & D4 & - & & \\
\hline & D5 & - & & \\
\hline \multirow[t]{5}{*}{5} & D1 & $1.43(1 ; 1.87)$ & \multirow[t]{5}{*}{$0.00 *$} & $\mathrm{D} 1 \times \mathrm{D} 20.01^{*}$ \\
\hline & D2 & $0.73(0.59 ; 0.86)$ & & $\mathrm{D} 1 \times \mathrm{D} 30.03 *$ \\
\hline & D3 & $0.98(0.85 ; 1.01)$ & & $\mathrm{D} 1 \times \mathrm{D} 40.61$ \\
\hline & D4 & $1.29(1.14 ; 1.6)$ & & $\mathrm{D} 1 \times \mathrm{D} 50.02 *$ \\
\hline & D5 & $0.7(0.67 ; 0.77)$ & & \\
\hline \multirow[t]{5}{*}{6} & D1 & $1.2(1.03 ; 1.39)$ & \multirow[t]{5}{*}{$0.00 *$} & $\mathrm{D} 1 \times \mathrm{D} 20.53$ \\
\hline & D2 & 1.13 (1.08; 1.28) & & $\mathrm{D} 1 \times \mathrm{D} 30.01 *$ \\
\hline & D3 & $2.32(2 ; 2.51)$ & & $\mathrm{D} 1 \times \mathrm{D} 40.01 *$ \\
\hline & D4 & $1.93(1.77 ; 2.05)$ & & $\mathrm{D} 1 \times \mathrm{D} 50.01^{*}$ \\
\hline & D5 & $1.5(1.33 ; 1.63)$ & & \\
\hline \multirow[t]{5}{*}{7} & D1 & $0.99(0.97 ; 1.22)$ & \multirow[t]{5}{*}{0.06} & - \\
\hline & D2 & $0.6(0.54 ; 0.85)$ & & \\
\hline & D3 & $0.98(0.82 ; 1.02)$ & & \\
\hline & D4 & $0.84(0.55 ; 1.06)$ & & \\
\hline & D5 & $0.69(0.37 ; 1.05)$ & & \\
\hline \multirow[t]{5}{*}{8} & D1 & $1.14(0.99 ; 1.51)$ & \multirow[t]{5}{*}{$0.00^{*}$} & $\mathrm{D} 1 \times \mathrm{D} 20.01 *$ \\
\hline & $\mathrm{D} 2$ & $2.29(1.94 ; 2.67)$ & & D1 × D3 $0.01 *$ \\
\hline & D3 & $3.67(2.87 ; 4.57)$ & & $\mathrm{D} 1 \times \mathrm{D} 40.01 *$ \\
\hline & D4 & 3.88 (3.44; 4.92) & & D1 x D5 $0.01 *$ \\
\hline & D5 & $5.52(4.48 ; 6.25)$ & & \\
\hline \multirow[t]{5}{*}{9} & D1 & $1.89(1.71 ; 2.11)$ & \multirow[t]{5}{*}{$0.01 *$} & $\mathrm{D} 1 \times \mathrm{D} 20.122$ \\
\hline & D2 & $1.47(1.38 ; 1.62)$ & & D1 × D3 0.73 \\
\hline & D3 & $1.96(1.5 ; 2.42)$ & & $\mathrm{D} 1 \times \mathrm{D} 40.29$ \\
\hline & D4 & $1.64(1.52 ; 1.88)$ & & $\mathrm{D} 1 \times \mathrm{D} 50.03 *$ \\
\hline & D5 & $1.08(0.82 ; 1.36)$ & & \\
\hline $10 * *$ & D1 & $1.14(0.64 ; 1.7)$ & 0.15 & - \\
\hline
\end{tabular}

Table 3 (continued)

\begin{tabular}{lllll}
\hline Participant & Day & Median (IQR) & $p$ value & Post hoc $p$ value \\
\hline \multirow{6}{*}{ D2 } & $0.65(0.4 ; 0.77)$ & & \\
D3 & - & & \\
D4 & - & & \\
& D5 & - & & \\
& D1 & $1.48(1.02 ; 1.62)$ & $0.01 *$ & D1 $1 \times$ D2 $0.01^{*}$ \\
D2 & $0.67(0.61 ; 0.87)$ & & D1 $\times$ D3 0.89 \\
D3 & $1.18(0.81 ; 2.4)$ & & D1 $\times$ D4 $0.09^{*}$ \\
D4 & $0.99(0.69 ; 1.31)$ & & D1 $\times$ D5 0.44 \\
D5 & $1.15(1.05 ; 1.59)$ & & \\
\hline
\end{tabular}

* Statistical difference at $p=0.05$.

**Participants completed D1 and D2.

$I Q R$ interquartile range.

inhibition) and increased short-interval intracortical facilitation reinforces the idea that a disruption of gammaaminobutyric acid-mediated cortical inhibitory effect is considered a key mechanism in the pathophysiology of chronic pain, and this seems to be especially present in neuropathic pain individuals. Otherwise, the restoration of intracortical inhibition through primary motor cortex stimulation (M1) [21] has been shown to be followed by a reduction in the intensity of some chronic pain groups [12, 22].

In a meta-analysis conducted by Metha et al., the use of tDCS in patients with SCINP has demonstrated to be a therapeutic option with a moderate effect size in reducing pain intensity, but with no sustained effect in most of the evaluated studies [20]. Chronicity of pain seems to be a determining factor in the quality of response $(<5$ years of pain as the most favorable response) [20]. In 2006, Fregni et al. also demonstrated a significant reduction in SCINP intensity when subjected to anodal stimulation of the primary motor cortex when compared to placebo. However, in this same study, it was possible to establish a negative correlation between the reduction in pain intensity and injury duration, indicating that there would be a narrow therapeutic window for the use of tDCS as adjuvant therapy in this population. This suggests that the possible mechanisms of action of neuromodulation would be more effective if applied earlier in the course of the SCI $[14,23]$. Maladaptive neuroplasticity affecting subcortical regions can also be evidenced days or years after spinal cord injury, often associated with reduced neuronal activity in these regions, with a significant correlation between the intensity of cortical reorganization and the intensity of neuropathic pain [7, 24-26]. This dysfunctional plasticity is related to thalamic, somatosensory, anatomical and functional changes, [27] and to the reorganization of the primary somatosensory cortex [26], although cortical reorganization and thalamic hypoactivity seem to be variable in 
Fig. 1 Individual $M E P$ quotient variation D1-D5. Participants in numerical ordereach symbol or dashed line represents the individual MEPquotient variation from D1 to D5.

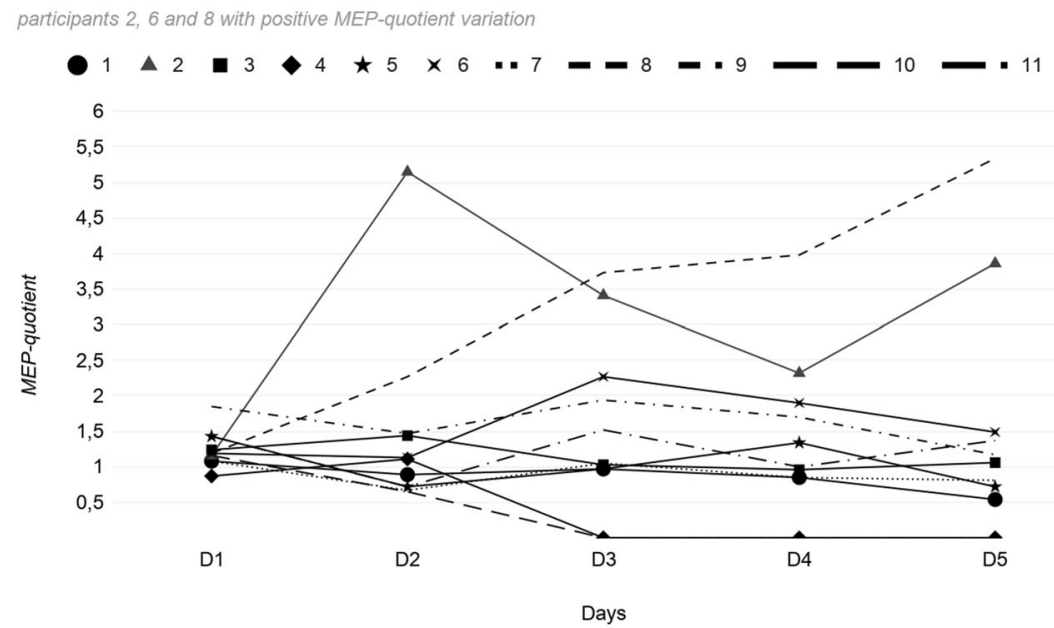

treatment of chronic neuropathic pain and the inability to alter the neurophysiological correlates of cortical plasticity within chronic SCINP individuals. Based on this series of cases and other clinical studies, the strength of recommendation of tDCS as an adjunctive treatment in individuals with chronic SCINP becomes weak.

From a clinical practice perspective, this is even more relevant, since its use may imply overtreatment burden and increased personal and institutional costs.

At last, chronic pain is a complex condition that involves both psychosocial and biological factors, with no hierarchy between these aspects in terms of its management. When establishing a therapeutic plan, it would be important for the clinician, when faced with the scientific evidence on the use of therapeutic neuromodulation, to recognize the limitations of these interventions and to consider multidimensional strategies of treatment for these individuals $[36,37]$.

\section{Data availability}

The datasets generated and/or analyzed during the current study are available from the corresponding author on reasonable request.

Acknowledgements The authors gratefully acknowledge Mrs. Mariana Vieira da Fonseca Ph.D. for the valuable statistical and methodological consulting, and Dr. Enio Alberto Comerlato and Dr. Denise Lima Medeiros de Melo for gently helping in acquisition of the neurophysiological parameters.

Author contributions VGC was responsible for designing the study protocol, writing the protocol, enrolling patients, extracting and analyzing data, interpreting results, updating reference lists, and drafting the final report. RLA was responsible for reviewing the study protocol, interpreting results and reviewing critically the final report for important intellectual content. RBB was responsible for reviewing the protocol, providing feedback on the final report and final approval of the version to be submitted. 


\section{Compliance with ethical standards}

Conflict of interest The authors declare that they have no conflict of interest.

Statement of ethics The present study was approved by the Associação das Pioneiras Sociais ethics committee (process number 2.357.788). We certify that all applicable institutional and governmental regulations concerning the ethical use of human health data were followed during the course of this research.

Publisher's note Springer Nature remains neutral with regard to jurisdictional claims in published maps and institutional affiliations.

\section{References}

1. Ackery A, Tator C, Krassioukov A. A global perspective on spinal cord injury epidemiology. J Neurotrauma. 2004;21:1355-70.

2. Dijkers M, Bryce T, Zanca J. Prevalence of chronic pain after traumatic spinal cord injury: a systematic review. J Rehabil Res Dev. 2009;46:13-29.

3. Nicholson BD. Evaluation and treatment of central pain syndromes. Neurology. 2004;62:S30-6.

4. Finnerup NB. Pain in patients with spinal cord injury. Pain. 2013;154:S71-6.

5. Dworkin RH, O'Connor AB, Backonja M, Farrar JT, Finnerup NB, Jensen TS, et al. Pharmacologic management of neuropathic pain: Evidence-based recommendations. Pain. 2007;132:237-51.

6. Pasero C. Pathophysiology of neuropathic pain. Pain Manag Nurs. 2004;5:3-8.

7. Kumru H, Vidal J, Kofler M, Portell E, Valls-Solé J. Alterations in excitatory and inhibitory brainstem interneuronal circuits after severe spinal cord injury. J Neurotrauma. 2010;27:721-8.

8. Costigan M, Scholz J, Woolf CJ. Neuropathic pain: a maladaptive response of the nervous system to damage. Annu Rev Neurosci. 2009;32:1-32.

9. Peyron R, Faillenot I, Mertens P, Laurent B, Garcia-Larrea L. Motor cortex stimulation in neuropathic pain. Correlations between analgesic effect and hemodynamic changes in the brain. A PET study. Neuroimage. 2007;34:310-21.

10. Parker RS, Lewis GN, Rice DA, Mcnair PJ. Is motor cortical excitability altered in people with chronic pain? A systematic review and meta-analysis. Brain Stimul. 2016;9:488-500.

11. Nitsche MA, Paulus W. Excitability changes induced in the human motor cortex by weak transcranial direct current stimulation. J Physiol. 2000;527:633-9.

12. Lefaucheur JP, Drouot X, Ménard-Lefaucheur I, Keravel Y, Nguyen JP, Menard-Lefaucheur I, et al. Motor cortex rTMS restores defective intracortical inhibition in chronic neuropathic pain. Neurology. 2006;67:1568-74.

13. Ngernyam N, Jensen MP, Arayawichanon P, Auvichayapat N, Tiamkao S, Janjarasjitt $S$, et al. The effects of transcranial direct current stimulation in patients with neuropathic pain from spinal cord injury. Clin Neurophysiol. 2015;126:382-90.

14. Fregni F, Boggio PS, Lima MC, Ferreira MJL, Wagner T, Rigonatti SP, et al. A sham-controlled, phase II trial of transcranial direct current stimulation for the treatment of central pain in traumatic spinal cord injury. Pain. 2006;122:197-209.

15. Nitsche MA, Paulus W. Sustained excitability elevations induced by transcranial DC motor cortex stimulation in humans. Neurology. 2001;57:1899-901.

16. Kirshblum SC, Burns SP, Biering-Sorensen F, Donovan W, Graves DE, Jha A, et al. International standards for neurological classification of spinal cord injury (Revised 2011) The authors are the members of the International Standards Committee of ASIA. J Spinal Cord Med. 2011;34:535-46.

17. Bryce TN, Biering-Sørensen F, Finnerup NB, Cardenas DD, Defrin R, Lundeberg T, et al. International spinal cord injury pain classification: Part I. Background and description. Spinal Cord. 2012;50:413-7.

18. Lefaucheur JP, Drouot X, Menard-Lefaucheur I, Zerah F, Bendib $\mathrm{B}$, Cesaro $\mathrm{P}$, et al. Neurogenic pain relief by repetitive transcranial magnetic cortical stimulation depends on the origin and the site of pain. J Neurol Neurosurg Psychiatry. 2004;75:612-6.

19. Nitsche MA, Liebetanz D, Antal A, Lang N, Tergau F, Paulus W. Modulation of cortical excitability by weak direct current stimulation-technical, safety and functional aspects. In: Suppl Clin Neurophysiol. 2003;56:255-76.

20. Mehta S, McIntyre A, Guy S, Teasell RW, Loh E. Effectiveness of transcranial direct current stimulation for the management of neuropathic pain after spinal cord injury: a meta-analysis. Spinal Cord. 2015;53:780-5.

21. Castillo Saavedra L, Mendonca M, Fregni F. Role of the primary motor cortex in the maintenance and treatment of pain in fibromyalgia. Med Hypotheses. 2014;83:332-6.

22. Botelho LM, Morales-Quezada L, Rozisky JR, Brietzke AP, Torres ILS, Deitos A, et al. A framework for understanding the relationship between descending pain modulation, motor corticospinal, and neuroplasticity regulation systems in chronic myofascial pain. Front Hum Neurosci. 2016;10:308-20.

23. Andrade DC, Borges I, Bravo GL, Bolognini N, Fregni F. Therapeutic time window of noninvasive brain stimulation for pain treatment: inhibition of maladaptive plasticity with early intervention. Exp Rev Med Devices. 2013;10:339-52.

24. Hsieh JC, Belfrage M, Stone-Elander S, Hansson P, Ingvar M. Central representation of chronic ongoing neuropathic pain studied by positron emission tomography. Pain. 1995;63:225-36.

25. Iadarola MJ, Max MB, Berman KF, Byas-Smith MG, Coghill RC, Gracely RH, et al. Unilateral decrease in thalamic activity observed with positron emission tomography in patients with chronic neuropathic pain. Pain. 1995;63:55-64.

26. Wrigley PJ, Press SR, Gustin SM, Macefield VG, Gandevia SC, Cousins MJ, et al. Neuropathic pain and primary somatosensory cortex reorganization following spinal cord injury. Pain. 2009;141:52-9.

27. Gustin SM, Wrigley PJ, Siddall PJ, Henderson LA. Brain anatomy changes associated with persistent neuropathic pain following spinal cord injury. Cereb Cortex. 2010;20:1409-19.

28. Moisset X, Bouhassira D. Brain imaging of neuropathic pain. Neuroimage. 2007;37:S80-8.

29. Wrigley PJ, Gustin SM, McIndoe LN, Chakiath RJ, Henderson LA, Siddall PJ. Longstanding neuropathic pain after spinal cord injury is refractory to transcranial direct current stimulation: a randomized controlled trial. Pain. 2013;154:2178-84.

30. Chagas AP, Monteiro M, Mazer V, Baltar A, Marques D, Carneiro $\mathrm{M}$, et al. Cortical excitability variability: Insights into biological and behavioral characteristics of healthy individuals. $\mathrm{J}$ Neurol Sci. 2018;390:172-7.

31. Fresnoza S, Paulus W, Nitsche MA, Kuo MF. Nonlinear dosedependent impact of D1 receptor activation on motor cortex plasticity in humans. J Neurosci. 2014;34:2744-53.

32. Stagg CJ, Best JG, Stephenson MC, O'Shea J, Wylezinska M, Kincses ZT, et al. Polarity-sensitive modulation of cortical neurotransmitters by transcranial stimulation. $\mathrm{J}$ Neurosci. 2009;29:5202-6.

33. McLaren ME, Nissim NR, Woods AJ. The effects of medication use in transcranial direct current stimulation: a brief review. Brain Stimul. 2018;11:52-8. 
34. Ziemann U, Lonnecker S, Steinhoff BJ, Paulus W. Effects of antiepileptic drugs on motor cortex fxcitabilitv in humans: a transcranial magnetk stimulation study. Ann Neurol. 1996;40:367-78.

35. Rizzo V, Quartarone A, Bagnato S, Battaglia F, Majorana G, Girlanda P. Modification of cortical excitability induced by gabapentin: a study by transcranial magnetic stimulation. Neurol Sci. 2001;22:229-32.
36. Burns AS, Delparte JJ, Ballantyne EC, Boschen KA. Evaluation of an interdisciplinary program for chronic pain after spinal cord injury. PMR. 2013;5:832-8.

37. Norrbrink Budh C, Kowalski J, Lundeberg T. A comprehensive pain management programme comprising educational, cognitive and behavioural interventions for neuropathic pain following spinal cord injury. J Rehabil Med. 2006;38:172-80. 